\title{
A thiazolothiazole containing multichromic polymer for glucose detection
}

\author{
S. Soylemez ${ }^{1 *}$, H. Z. Kaya ${ }^{2}$, Y. A. Udum ${ }^{3}$, L. Toppare $2,4,5,6$ \\ ${ }^{1}$ Department of Chemistry, Ordu University, 52200 Ordu, Turkey \\ ${ }^{2}$ Department of the Center for Solar Energy Research and Application (GUNAM), Middle East Technical University, \\ 06800 Ankara, Turkey \\ ${ }^{3}$ Technical Sciences Vocational School, Gazi University, 06374 Ankara, Turkey \\ ${ }^{4}$ Department of Chemistry, Middle East Technical University, 06800 Ankara, Turkey \\ ${ }^{5}$ Department of Biotechnology, Middle East Technical University, 06800 Ankara, Turkey \\ ${ }^{6}$ Department of Polymer Science and Technology, Middle East Technical University, 06800 Ankara, Turkey
}

Received 30 January 2019; accepted in revised form 4 May 2019

\begin{abstract}
Donor-acceptor (DA) type monomers namely 2,5-di(thiophen-2-yl)thiazolo[5,4-d]thiazole (TTzTh) and 2,5-bis(3methylthiophen-2-yl)thiazolo[5,4-d]thiazole (TTzMTh) were synthesized and their electrochemical and optoelectronic properties were investigated in detail. The spectro-electrochemical analysis showed that the alkyl chain substitution results in a shift in the onset of the $\pi-\pi^{*}$ transition towards longer wavelengths. Depending on the donor substituents, the polymers exhibited optical band gaps 1.65 and $1.85 \mathrm{eV}$ for PTTzTh and PTTzMTh, respectively. Electrochromic studies revealed that both polymers are p-dopable and multichromic. Moreover, polymer of TTzTh (PTTzTh) has been used for the development of a glucose biosensor. Glucose oxidase (GOx) was anchored on a graphite electrode which was previously modified with a film of the conjugated polymer, PTTzTh by electropolymerization. Such a sensor showed a wide linear range (0.05$2.0 \mathrm{mM})$, good sensitivity $\left(36.32 \mu \mathrm{A} /\left(\mathrm{mM} \cdot \mathrm{cm}^{2}\right)\right.$ and low limit of detection (LOD) $(0.075 \mathrm{mM})$ under formerly optimized conditions. Moreover, the accuracy of the biosensor was successfully tested using two different beverages to detect glucose. Electrochemical characterizations of the polymers and their biosensor application were investigated for the first time in this work.
\end{abstract}

Keywords: polymer synthesis, molecular engineering, amperometric glucose biosensor, thiazolothiazole bearing conjugated polymer, electrochromism

\section{Introduction}

Glucose detection is a very important issue all over the world for diagnosis and prevention of diabetes mellitus. High levels of glucose in the blood cause certain damages in human body [1]. To control this level by monitoring glucose amount in blood is possible using many of the traditional methods. Electrochemical biosensors are very popular tools to detect glucose quantitatively [2]. Such biosensors have some advantages over other methods. These do not require high skilled personnel, complicated sample preparation or expensive characterizations. Enzymebased diagnostic techniques are a rising issue due to their reasonable potential in wide range of applications. However, one crucial issue to construct enzyme based systems is anchoring of biomolecules on the electrode surface. A number of relevant literature reported that different materials have been used for enzyme immobilization [3-7].

The charming properties of conjugated polymeric materials led scientists to explore the opportunities of utilizing them in biosensor constructions. Conjugated 
polymer based biosensors are recognized to be a next generation building architecture for constructing highly sensitive and fast bio-sensing systems. The use of conjugated polymers as great immobilization architectures for biomolecules motivates the development of efficient biosensors [8-10]. The designed surface allows easy detection of analyte via providing the best conformational structure for biomolecule. It also enables easy preparation to reduce analysis time. In this regard, conjugated polymers remain within the top materials used in development of sensors and nanodevices. In literature, several studies have summarized utilizing conjugated polymers in glucose biosensor applications [7,11-14]. Despite the fact that a number of supporting materials are needed to construct a well-equipment glucose biosensor, in this work promising biosensor parameters and wide linear range were obtained via using a pristine conjugated polymer on the electrode surface. Moreover, the unit thiazolothiazole (TTz) has been shown to be a promising material for optoelectronics with extended $\pi$ electron system with a rigid and coplanar backbone and strong $\pi$-stacking $[15,16]$. Due to its high charge carrier mobility, it is a preferred material to be used as a subunit in p-type conjugated polymers especially for polymer solar cells and organic field effect transistor applications $[17,18]$. Recently, TTz containing structures exhibited strong fluorescence and viologen-like reversible electrochromism with high quantum yields [19].

This paper describes the synthesis, characterization and electropolymerization of two thiazolothiazoles containing donor-acceptor-donor type monomers with thiophene and 3-methylthiophene as the donor units. Optical and electrochromic properties of the corresponding polymers were investigated using cyclic voltammetry and in situ spectro-electrochemical techniques. Polymers showed multichromic properties under applied voltages with low band-gap values. Furthermore, we fabricated a glucose biosensor utilizing the electropolymerization of TTzTh. After electropolymerization of TTzTh, glucose oxidase (GOx) was immobilized on the polymer coated surface and further used for glucose detection in beverages. GOx is a type of oxide-reductase enzyme that contains flavin adenine dinucleotide (FAD) redox center. During the enzymatic reaction, GOx catalyzes the oxidation of glucose to D-glucono-1,5-lactone and hydrogen peroxide, which is formed in the action of molecular oxygen as an electron acceptor
[20]. The level of the oxygen consumption was followed at $-0.7 \mathrm{~V}$ vs Ag wire reference electrode by amperometric detection technique. The bio-catalytic reaction at a working electrode of biosensor associates with the reduction of the FAD by reaction with $\beta$-D-glucose. In the following step, $\mathrm{GOx}-\mathrm{FADH}_{2}$ is oxidized by the final electron acceptor, molecular oxygen $\left(\mathrm{O}_{2}\right)$. During this process, the reduced form $-\mathrm{GOx}-\mathrm{FADH}_{2}$-is regenerated back into oxidized form -GOx-FAD, and in addition $\mathrm{H}_{2} \mathrm{O}_{2}$ is formed [4].

\section{Experimental}

\subsection{Materials and equipment}

Chemicals used in organic synthesis were taken from Sigma Aldrich and directly used without further purification. ${ }^{1} \mathrm{H}$ NMR (proton nuclear magnetic resonance) chemical shifts were given in ppm downfield from tetramethylsilane (TMS) recorded in $\mathrm{CDCl}_{3}$ solvent on Bruker Spectrospin Avance DPX-400 Spectrometer. High resolution mass analyses (HRMS) of the monomers were recorded on Waters SYNAPT MS System to confirm the monomer synthesis. Electrochemical polymerization of monomers was carried out in a three-electrode cell containing ITO (indium tin oxide) coated glass slide $\left(12 \Omega / \mathrm{cm}^{2}\right)$ as the working electrode, a platinum wire and $\mathrm{Ag}$ wire as the counter and pseudo-reference electrodes, respectively. The same reference and counter electrodes were also used in biosensor measurements. Electrochemical studies of the polymers were performed with a Gamry Reference 600 potentiostat. Spectroelectrochemistry and kinetic characterizations of the polymer film were achieved with Jasco V-770 UV-VisNIR (ultraviolet - visible - near infrared) spectrophotometer coupled with Solartron 1285 Potentiostat. Minolta CS-100 spectrophotometer was used to determine the three attributes $(L, a, b)$ of film colors as defined by Commission Internationale de lé Clairage (CIE).

Glucose oxidase (GOx, $\beta$-D-glucose: oxygen 1-oxidoreductase, EC 1.1.3.4, 17300 units/g solid) from $A$. Niger, D-glucose, glutaraldehyde (GA, Grade II 25\% in $\mathrm{H}_{2} \mathrm{O}$ ), $\mathrm{NaClO}_{4}$ and $\mathrm{LiClO}_{4}$ were all obtained from Sigma-Aldrich and were used without further purification. Phosphate buffer solution was prepared using $0.025 \mathrm{M} \mathrm{Na}_{2} \mathrm{HPO}_{4}$ (Fisher Scientific Company) and $0.025 \mathrm{M} \mathrm{NaH}_{2} \mathrm{PO}_{4}$ (Fisher Scientific Company). Glucose solution $(0.1 \mathrm{M}$, as a substrate) was prepared by dissolving $0.18 \mathrm{~g}$ of glucose in $10 \mathrm{ml} \mathrm{pH}$ 7.0 phosphate buffer solution. 
Electrochemical measurements were carried out at room temperature setting a PalmSens potentiostat (PalmSens, Houten, The Netherlands). A three electrode cell with a graphite electrode (Ringsdorff Werke $\mathrm{GmbH}$, Bonn, Germany, type RW001, 3.05 mm diameter and $13 \%$ porosity) was used as a working electrode. The data were given as the average of three measurements and standard deviations were recorded as \pm SD. Amperometric measurement studies were performed at room temperature in a $10 \mathrm{ml}$ electrochemical cell equipped with three electrode configurations. This measurement was recorded in a reaction cell filled with phosphate buffer solution $(\mathrm{pH}$ 7.0) under mild stirring by applying $-0.7 \mathrm{~V}$ constant potential. After each consecutive measurement, new buffer solution was used. When the baseline current reached to equilibrium, certain amount of glucose was added into the reaction medium and equilibrium was established again. The current change was followed for recording biosensor responses.

\subsection{Synthesis of monomers}

Synthesis of the monomers was performed in a single step reaction by condensation of dithiooxamide with related aldehydes. Obtained monomers were electrochemically polymerized on ITO surfaces, described in next section in detail. Synthetic procedure and the NMR spectra of the monomers are depicted in Figure 1.

\subsubsection{Synthesis of TTzTh}

TTzTh (1b) monomer was synthesized using a described procedure from literature [21]. A solution of thiophene-2-carbaldehyde ( $2 \mathrm{~g}, 17.8 \mathrm{mmol})$ and dithiooxamide ( $1 \mathrm{~g}, 8.9 \mathrm{mmol})$ in $N, N$-dimethylformamide (DMF) $(30 \mathrm{ml})$ was refluxed for $3 \mathrm{~h}$. After cooling the mixture to room temperature, it was poured into $100 \mathrm{ml}$ of cold water and the precipitate was extracted by dichloromethane. Solvent was dried over magnesium sulphate and it was evaporated under vacuum. The residue was recrystallized using dichloromethane/methanol system to obtain brownish yellow crystals with a yield of $26 \%$. ${ }^{1} \mathrm{H}$ NMR $\left(400 \mathrm{MHz}, \mathrm{CDCl}_{3}, \mathrm{ppm}\right) \delta: 7.50(\mathrm{~d}, 2 \mathrm{H}), 7.40(\mathrm{~d}$, $2 \mathrm{H}), 7.05(\mathrm{~m}, 2 \mathrm{H})$. HRMS (ES ${ }^{+}$) for $\mathrm{C}_{12} \mathrm{H}_{6} \mathrm{~N}_{2} \mathrm{~S}_{4}$, calculated 306.4492 found 306.4482 .

\subsubsection{Synthesis of TTzMTh}

Same procedure was applied in the synthesis of TTzMTh (2b) using 3-methylthiophene-2-carbaldehyde $(5 \mathrm{~g}, 39.6 \mathrm{mmol})$ and dithiooxamide $(2.4 \mathrm{~g}$, $19.8 \mathrm{mmol}$ ) as the reactants in $50 \mathrm{ml}$ of DMF. After recrystallization a red solid was obtained with a yield of $12 \%$. ${ }^{1} \mathrm{H}$ NMR (400 MHz, $\left.\mathrm{CDCl}_{3}, \mathrm{ppm}\right) \delta: 7.33$ (d, 2H), 7.06 (d, 2H), 2.30 (s, 6H). HRMS $\left(\mathrm{ES}^{+}\right)$for $\mathrm{C}_{14} \mathrm{H}_{10} \mathrm{~N}_{2} \mathrm{~S}_{4}$, calculated 334.9805 found 334.9821 .

\section{Results and discussion}

\subsection{Electrochemical polymerization of the monomers}

Electrochemical polymerization of polymers (PTTzTh and PTTzMTh) were performed using conventional three-electrode setup via cyclic voltammetry in a mixed solvent system (acetonitrile/dichloromethane: 95:5) containing $0.1 \mathrm{M} \mathrm{NaClO}_{4} / \mathrm{LiClO}_{4}$ as the supporting electrolyte. Polymers were deposited on ITO

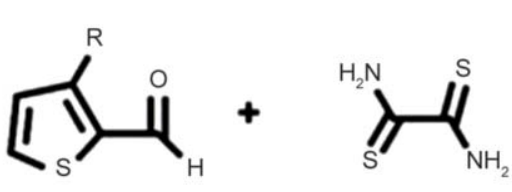

a) $\mathrm{R}: \underset{\mathrm{CH}_{3}}{\mathrm{H}}(1 \mathrm{a})$

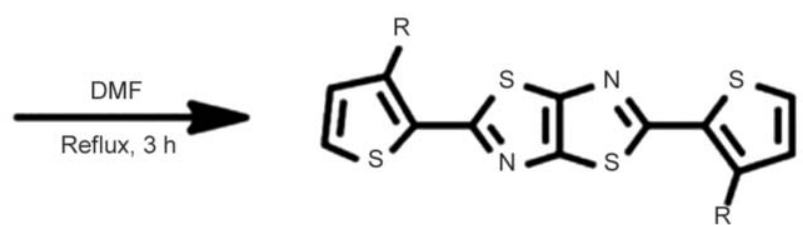

$\mathrm{R}: \underset{\mathrm{CH}}{\mathrm{H}}(1 \mathrm{~b})$
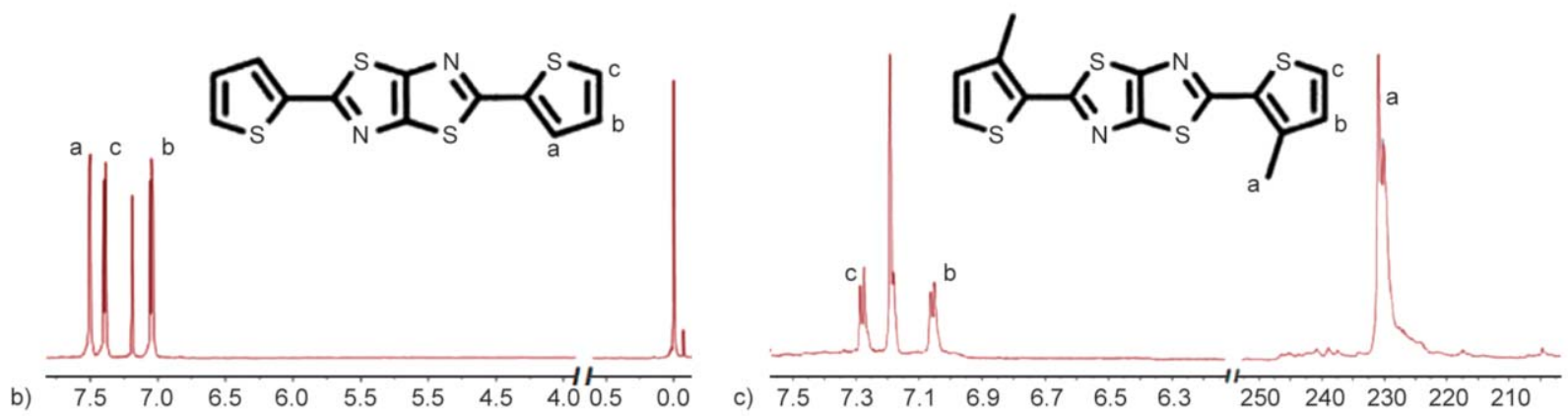

Figure 1. (a) Synthetic route of monomers, (b) NMR spectrum of $\mathbf{1 b}$ and (c) NMR spectrum of $\mathbf{2} \mathbf{b}$. 
electrodes with a scan rate of $100 \mathrm{mV} / \mathrm{s}$ with a platinum wire as the counter and a silver wire as the reference electrodes.

As depicted in Figure 2, first cycles of the CV scans represent the electrochemical behaviour of the monomers. The initial oxidation of the TTzMTh was observed at $+1.0 \mathrm{~V}$, which is lower than that of TTzTh $(+1.38 \mathrm{~V})$ owing to the electron donating behaviour of the methyl group on the thiophene ring. Thiazolothiazole containing PTTzMTh showed lower monomer oxidation potential than its analogue reported previously, which shows that TTz is a more suitable acceptor [22]. The formation of the new redox couples and increase in the current densities as the repetitive cycles indicate the formation of the polymers on ITO surfaces $[23,24]$. The CV curve of the PTTzTh has a doping/de-doping couple located at $+0.80 \mathrm{~V} /+0.65 \mathrm{~V}$. The de-doping peak shifts to a lower potential with increasing the number of cycles due to the increased conjugation on the electrode surface. Same behaviour was observed for PTTzMTh, de-doping peak appeared at $+0.55 \mathrm{~V}$. The corresponding doping peak was observed at about $+0.78 \mathrm{~V}$ [25]. Additionally, a broader redox couple was observed for PTTzMTh than that of PTTzTh, which can be attributed to the formation of the conjugated chains with different chain length leading in situ transition of several electroactive species on the surface [2628]. After 10 cycles of electrochemical polymerization, resulting polymers showed the shades of the colour brown in their neutral states.

\subsection{Spectroelectrochemistry}

Investigation of the optoelectronic properties of the polymer films were performed by spectroelectro-

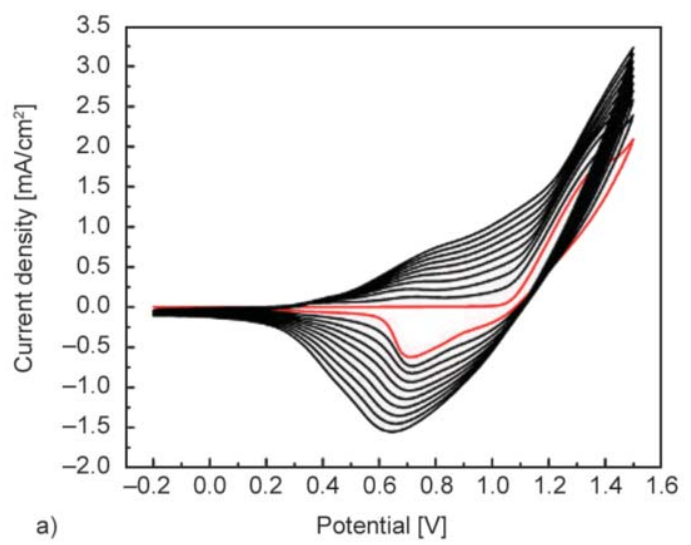

chemistry. Absorption spectra of the PTTzTh and PTTzMTh were recorded while applying potential in a monomer free electrolytic medium containing $0.1 \mathrm{M} \mathrm{NaClO}_{4} / \mathrm{LiClO}_{4}$. Optical band gaps of these D-A-D type electrochromic polymers were calculated from the onset of the low energy regions of the neutral state absorptions. Band gap value of PTTzTh was calculated as $1.65 \mathrm{eV}$ using equation $E_{\mathrm{g}}=$ $1241 / \lambda_{\text {onset. }}$ Presence of the alkyl chains at the $\beta$-position of thiophene moiety influences the electronic structure of the polymer. The alkyl substituents on the polymer cause steric hindrance, resulting in less order and less conjugation and an increase in polymer band gap. Hence PTTzMTh exhibits a larger Eg value $(1.85 \mathrm{eV})$ than that of PTTzTh calculated by the onset of the $\pi-\pi^{*}$ transition shifts.

The effect of the electron donating methyl group on thiophene ring was also observed during the stepwise oxidation of the semiconducting polymers to achieve different electronic levels for electrochromic characterization. Polymers have absorption maxima at $440 \mathrm{~nm}$ (PTTzTh) and $460 \mathrm{~nm}($ PTTzMTh) in their neutral states. As seen in Figure 3, compared to PTTzTh, PTTzMTh has $20 \mathrm{~nm}$ red shift for the maximum absorption wavelength due to the strength of the MTh donor unit. Upon oxidation of the polymer films, the intensity of their neutral state absorption bands decreases and two new peaks are generated. The evolution of the polaronic band at around $710 \mathrm{~nm}$ for PTTzTh leads to a dual-band absorption in the visible region. This causes the change colors of the polymer film into the hues of green. In the case of PTTzMTh, similar band generation was observed at $654 \mathrm{~nm}$ which is not well separated from the band at $460 \mathrm{~m}$ and showed grey color in its intermediate

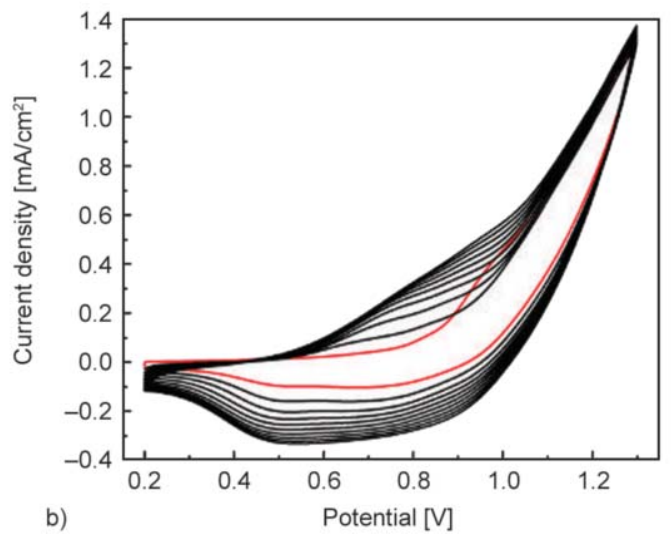

Figure 2. Cyclic voltammograms of $1 \cdot 10^{-3} \mathrm{M}$ PTTzTh (a) and PTTzMTh (b) in $0.1 \mathrm{M} \mathrm{LiClO}_{4} / \mathrm{NaClO}_{4} / \mathrm{DCM} / \mathrm{ACN}$. Potential scan rate: $100 \mathrm{mV} / \mathrm{s}$. 

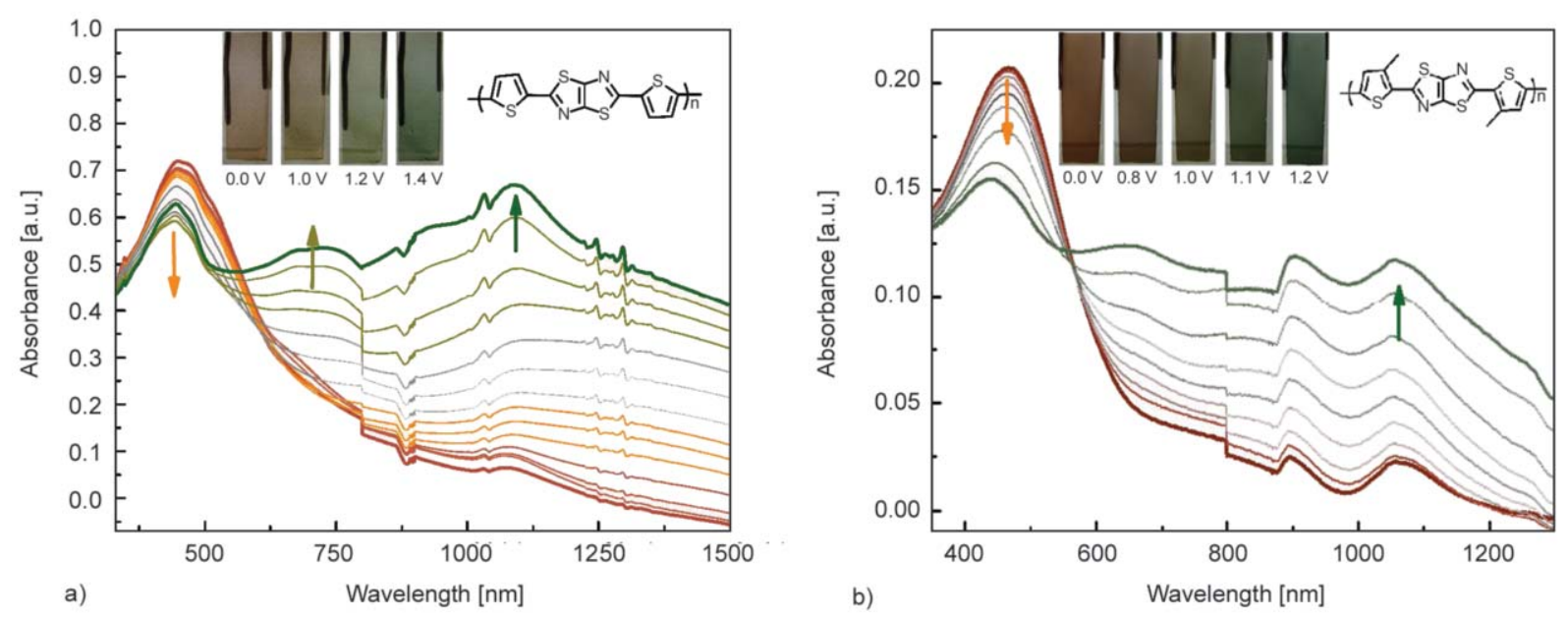

Figure 3. UV-Vis-NIR absorption spectra of (a) PTTzTh and (b) PTTzMTh film under various applied potentials.

level. During further oxidation of both polymers, bipolaronic bands were observed which are located at $1080 \mathrm{~nm}$ for both PTTzTh and PTTzMTh [29]. The observed colors during oxidative $p$-doping of the polymer films were indexed according to Commission international de I'Eclairage (CIE) and listed in Table 1. Defined as luminance $L^{*}$, hue $a^{*}$, and saturation $b^{*}$ values are used to gain a scientific understanding for electrochromic applications. $L^{*}$ is a measure of brightness and $a^{*}$ and $b^{*}$ are related to the redgreen and yellow-blue saturation, respectively [25].

\subsection{Kinetic studies}

Optical contrast and the response time are two crucial parameters for electrochromics, which are defined as the percent transmittance change $(\Delta T \%)$ between two ultimate states of a polymer film and the time required to observe of this change, respectively. Double step chronoamperometry method was applied to define these parameters by a spectrophotometer. Polymer films were deposited on ITO glasses under same conditions used for spectroelectrochemistry. The potentials were switched between -0.2 and $1.4 \mathrm{~V}$ for PTTzTh in $5 \mathrm{~s}$ time intervals whereas +0.2 and $+1.2 \mathrm{~V}$ were applied for PTTzMTh. Switching times were calculated considering $95 \%$ of the transmittance change that a naked eye can not realize beyond [30]. Optical change of the PTTzTh were found as 14\% at $440 \mathrm{~nm}$ with switching times of $2.36 \mathrm{~s}$ for oxidation and $0.35 \mathrm{~s}$ for reduction (Figure 4). Replacing the thiophene unit with methylthiophene resulted in a higher optical contrast value in visible region for the PTTzMTh which is found to be $30 \%$ at $460 \mathrm{~nm}$ (Figure 5). Additionally, a faster switching time ( $0.75 \mathrm{~s})$ was determined for the oxidation of PTTzMTh in this region. PTTzTh showed $11 \%$ optical contrast in its polaronic region. Highest optical contrast value $(48 \%)$ was obtained for PTTzTh at NIR region and the response times were calculated as $0.7 \mathrm{~s}$ from the reduced to oxidized state and $3.05 \mathrm{~s}$ from oxidized to reduced state. Interestingly, the optical contrast value of PTTzMTh was calculated as only $18 \%$ which decreases after 50 seconds of switching showing PTTzTh is more suitable for NIR applications. All electrochromic parameters for polymers PTTzTh and PTTzMTh were summarized in Table 2, which are

Table 1. Color coordinates for PTTzTh and PTTzMTh film as a function of the applied potentials.

\begin{tabular}{|c|c|c|c|c|}
\hline Polymers & E, vs. Ag wire & $L^{*}$ & $a^{*}$ & $b^{*}$ \\
\hline & $0.0 \mathrm{~V}$ & 43.671 & 2.345 & 16.530 \\
\hline & $1.0 \mathrm{~V}$ & 38.342 & -1.451 & 19.245 \\
\hline & $1.2 \mathrm{~V}$ & 42.996 & -8.973 & 18.273 \\
\hline PTT & $1.4 \mathrm{~V}$ & 36.144 & -12.109 & 11.653 \\
\hline \multirow[b]{5}{*}{ PTTzMTh } & $0.0 \mathrm{~V}$ & 27.858 & 9.073 & 17.652 \\
\hline & $0.8 \mathrm{~V}$ & 31.553 & 0.919 & 14.890 \\
\hline & $1.0 \mathrm{~V}$ & 33.057 & -2.058 & 14.395 \\
\hline & $1.1 \mathrm{~V}$ & 28.271 & -3.730 & 8.222 \\
\hline & $1.2 \mathrm{~V}$ & 27.855 & -10.535 & 7.479 \\
\hline
\end{tabular}



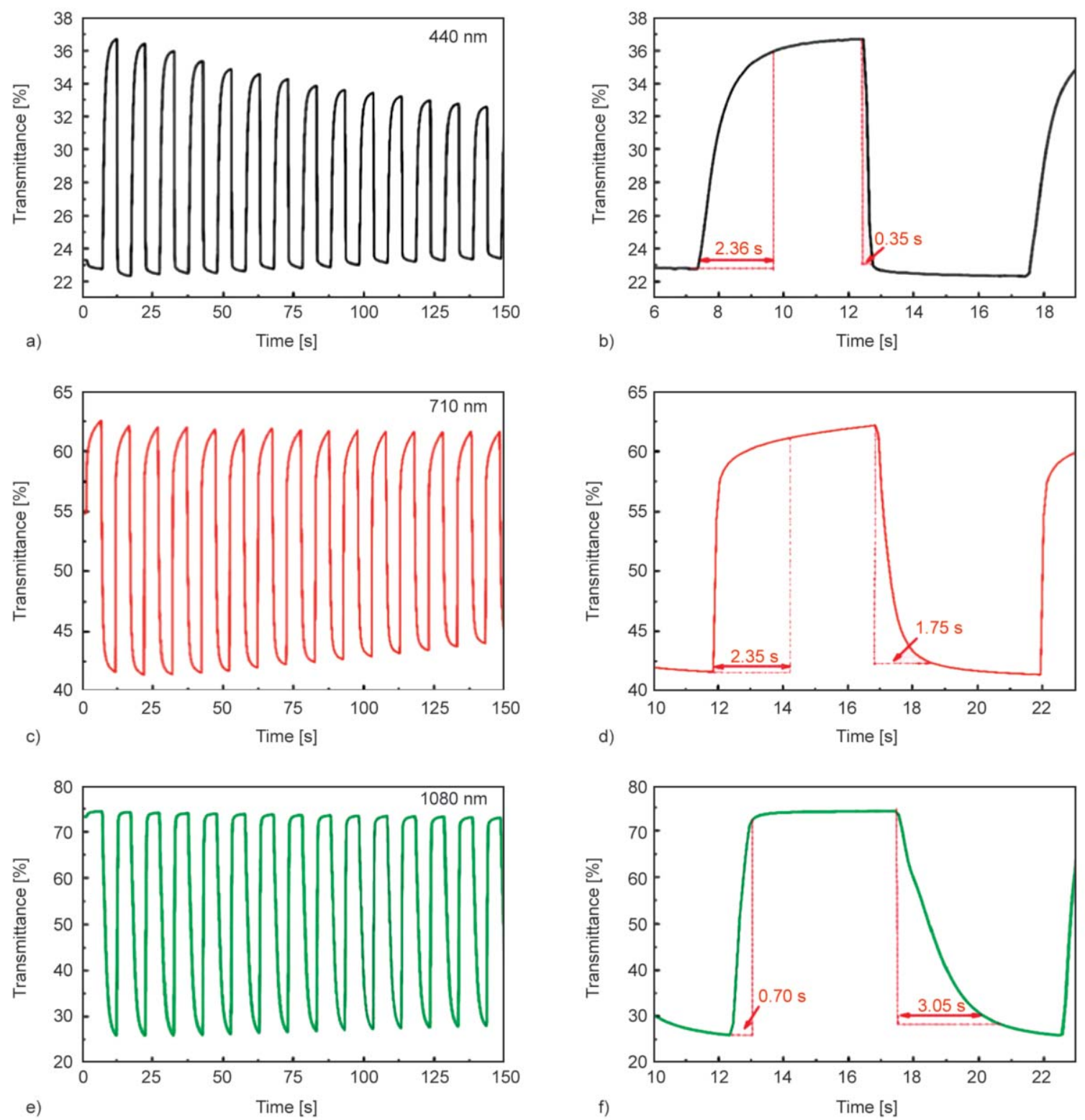

Figure 4. In situ transmittance responses and electrochromic switching for PTTzTh monitored at $440 \mathrm{~nm}$ (a and b), $710 \mathrm{~nm}$ (c and d), $1080 \mathrm{~nm}$ (e and $\mathrm{f}$ ) for the residence time of $5 \mathrm{~s}$.

comparable to those for electrochromic polymers [31, 32].

To investigate the switching stability of the polymer films, PTTzTh and PTTzMTh were deposited on ITO glass electrodes. Cycling voltammetry technique were used while switching between their doped and neutral states in $\mathrm{LiClO}_{4} / \mathrm{NaClO}_{4} / \mathrm{DCM} / \mathrm{ACN}$ electrolyte/solvent couple. PTTzTh and PTTzMTh polymer films retained 49 and $34 \%$ of their activities, respectively after 100 repetitive cycles.
Table 2. Electrochemical and optical properties of the polymers.

\begin{tabular}{|ll|c|c|}
\hline & & PTTzTh & PTTzMTh \\
\hline$E_{\text {monomer, ox }}$ & {$[\mathrm{V}]$} & +1.38 & +1.00 \\
\hline$E_{\text {doped, ox }}$ & {$[\mathrm{V}]$} & +0.80 & +0.78 \\
\hline$E_{\text {dedoped, ox }}$ & {$[\mathrm{V}]$} & +0.65 & +0.55 \\
\hline$E_{\mathrm{g}}{ }^{\text {op }}$ & {$[\mathrm{eV}]$} & 1.65 & 1.85 \\
\hline$\lambda_{\max }$ & {$[\mathrm{nm}]$} & $440 / 710 / 1080$ & $460 / 1080$ \\
\hline \multicolumn{2}{|l|}{ Optical contrasts $[\%]$} & $14 / 21 / 50$ & $30 / 18$ \\
\hline \multicolumn{2}{|l|}{ Switching times } & $2.36 / 0.35$ & $0.75 / 1.30$ \\
& $2.35 / 1.75$ & $0.50 / 1.10$ \\
& & \\
\hline
\end{tabular}



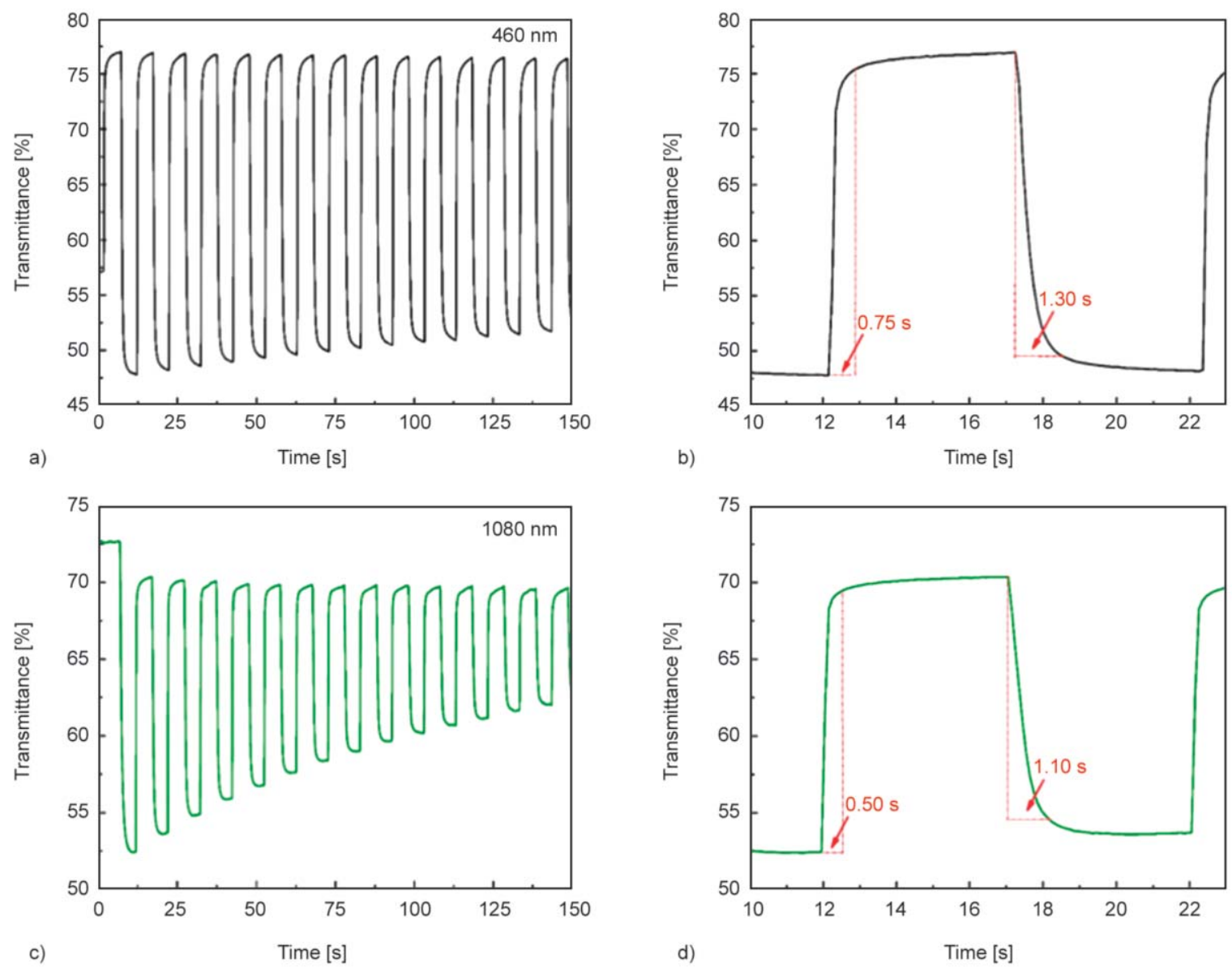

Figure 5. In situ transmittance response and electrochromic switching for PTTzMTh monitored at $460 \mathrm{~nm}$ (a and b), $1080 \mathrm{~nm}(\mathrm{c}$ and $\mathrm{d})$ for the switching time of $5 \mathrm{~s}$.

\subsection{Biosensor application}

\subsubsection{Preparation of the biosensor}

For biosensor applications, first of all graphite electrodes were cleaned using emery paper and washed with distilled water. Electropolymerization of TTzTh monomer was performed via cyclic voltammetry on a cleaned graphite electrode in a cell (ACN: DCM/ 95:5) containing $0.1 \mathrm{M} \mathrm{NaClO}_{4} / \mathrm{LiClO}_{4}$ as the supporting electrolyte. PTTzTh was deposited on graphite electrode scanning the potential between -0.2 and $1.5 \mathrm{~V}$ potentials with a scan rate of $100 \mathrm{mV} \cdot \mathrm{s}^{-1}$ for 30 cycles. After electrochemical polymerization process, the polymer coated graphite electrode was rinsed with distilled water to remove impurities. Then, $1.0 \mathrm{mg}$ GOx was dissolved in $10.0 \mu 1$ of sodium phosphate buffer ( $\mathrm{pH} 7.0,50 \mathrm{mM}$ ) and was cast onto the conducting polymer coated electrode surface and then, GA solution $(5.0 \mu 1,1 \%)$ was spread over the surface. The electrode was allowed at room temperature for drying. Figure 6 represents the procedure of the construction of newly designed amperometric glucose biosensor.

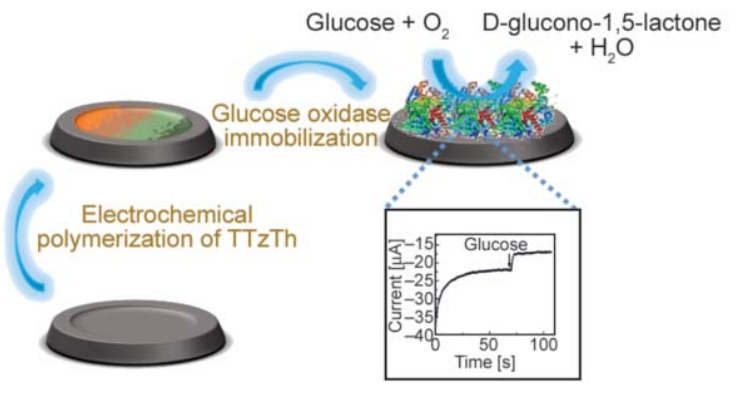

Figure 6. Schematic representation of the glucose biosensor.

\subsubsection{Characterization of the biosensor}

Under optimum conditions, biosensor behaviours were evaluated. Biosensor responses were recorded for varying glucose concentrations and a calibration curve was drawn (Figure 7). Linear responses were obtained for glucose ranging from 0.05 to $2.0 \mathrm{mM}$ using the equation $y=2.3569 x+0.2456\left(R^{2}=0.995\right)$ by PTTzTh/GOx biosensor. Also, sensitivity and the limit of detection (LOD) values were calculated to be $36.32 \mu \mathrm{A} /\left(\mathrm{mM} \cdot \mathrm{cm}^{2}\right)$ and $0.075 \mathrm{mM}$ by setting the intercept of the linear range of the calibration curve 


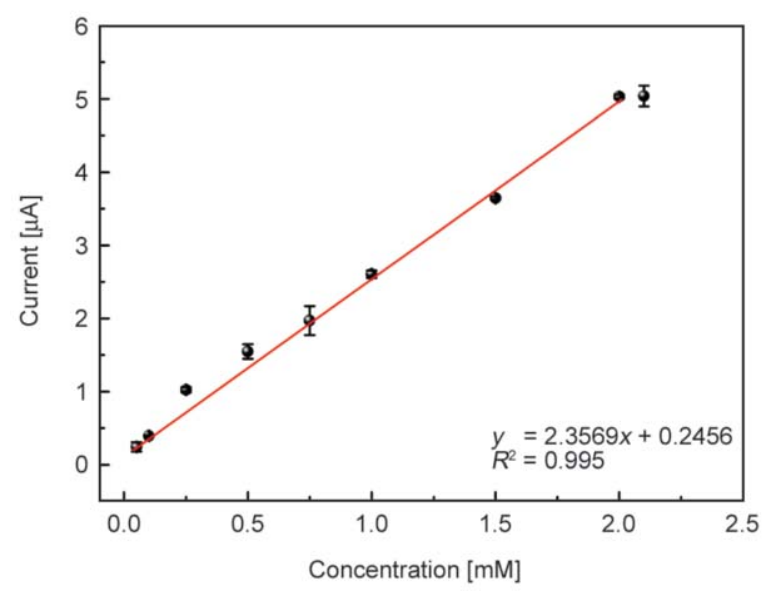

Figure 7. Calibration curve for glucose (in $50 \mathrm{mM}$ phosphate buffer $\mathrm{pH} 7.0$ buffer, $25^{\circ} \mathrm{C},-0.7 \mathrm{~V}$ ).

to zero using the $S / N$ (signal-to-noise ratio) $=3$ criterion. Compared to values reported in the literature, the ones for the proposed sensing system have very good sensor characteristics. Although there are a number of examples related to conjugated polymerbased sensors in literature, in most cases these systems require combination with other materials in order to obtain promising biosensor characteristics. However, in the proposed system, the electrochemically polymerized (TTzTh) monomer showed superior parameters since it is the only material on the electrode surface without requiring combination with anything else where it was used for the first time in a glucose biosensor application. For example, Periasamy et al. [33] designed a surface based on gelatinmultiwalled carbon nanotube (GCNT) modified glassy carbon electrode (GCE) and GOx was immobilized on the modified surface with the help of glutaraldehyde chemistry. GCNT/GOx/GAD biosensor showed sensitivity of $2.47 \mu \mathrm{A} /\left(\mathrm{mM} \cdot \mathrm{cm}^{2}\right)$ for glucose at the composite film. Wan et al. [34]. demonstrated that an amperometric glucose biosensor prepared using polyaniline (PANI) and chitosan-coupled carbon nanotubes (CS-CNTs) on a gold electrode (the Au-g-PANI-c-(CS-CNTs)-GOD). The resulting Aug-PANI-c-(CS-CNTs)-GOD biosensor exhibited $21 \mu \mathrm{A} /\left(\mathrm{mM} \cdot \mathrm{cm}^{2}\right)$ sensitivity. Similarly, glucose oxidase was immobilized in a layer of polypyrrole (PPY) by electropolymerization of the corresponding monomer on platinum electrode modified by Prussian Blue (PB). The related sensor showed a sensitivity value of $1.3 \mu \mathrm{A} /\left(\mathrm{mM} \cdot \mathrm{cm}^{2}\right)$ [35]. In another study, laponite/chitosan/GOx biosensor system was designed and this sensing system exhibited $33.9 \mu \mathrm{A} /\left(\mathrm{mM} \cdot \mathrm{cm}^{2}\right)$ sensitivity value with a $10^{-3}$
$5 \cdot 10^{-2} \mathrm{mM}$ linear range [36]. Additionally, poly (adamantanepyrrole)/SWCNT/B-cyclodextrin/GOx was constructed by Holzinger et al. [37]. In this study, sensitivity and $K_{\mathrm{M}}^{\text {app }}$ values were reported as $31.02 \mu \mathrm{A} /\left(\mathrm{mM} \cdot \mathrm{cm}^{2}\right)$ and $5.0 \mathrm{mM}$, respectively. Furthermore, prussian blue (PB) was combined with the poly(o-aminophenol) film to develop the glucose biosensor and sensitivity and $K_{\mathrm{M}}^{\mathrm{app}}$ values were calculated as $24.00 \mu \mathrm{A} /\left(\mathrm{mM} \cdot \mathrm{cm}^{2}\right)$ and $10.5 \mathrm{mM}$ [38]. Bienzymatic glucose biosensor was also carried out by Alim et al. [39]. According to the experimental results, such a sensor displayed good analytical characteristics. However, a considerable drawback of the system is that it requires a higher potential for hydrogen peroxide oxidation. In another work, a platinum/reduced graphene oxide/poly(3-aminobenzoic acid) $(\mathrm{Pt} / \mathrm{rGO} / \mathrm{P} 3 \mathrm{ABA})$ nanocomposite film was deposited on a screen-printed carbon electrode (SPCE) and its application in the development of amperometric glucose biosensor was demonstrated [40]. The proposed platform showed sensitivity and $K_{\mathrm{M}}$ values as $22.01 \mu \mathrm{A} /\left(\mathrm{mM} \cdot \mathrm{cm}^{2}\right)$ and $3.54 \mathrm{mM}$, respectively. Moreover, $\mathrm{SiO}_{2}\left(\mathrm{LuPc}_{2}\right) \mathrm{PANI}(\mathrm{PVIA})-\mathrm{CNB}$ sensor was prepared to detect glucose in real samples (juice and serum). The sensitivity value is recorded as $38.53 \mu \mathrm{A} /\left(\mathrm{mM} \cdot \mathrm{cm}^{2}\right)$ for fabricated sensor under optimized conditions [41]. A polypyrrole (PPy)-based bilayer amperometric glucose biosensor integrated with a perm-selective layer has been developed for detection of glucose [42]. The $K_{\mathrm{M}}$ and sensitivity values were also determined as $8.4 \mathrm{mM}$ and $3.5 \mu \mathrm{A} /\left(\mathrm{mM} \cdot \mathrm{cm}^{2}\right)$. Similarly, enzyme-based glucose biosensor was fabricated by Campbell et al. [43]. To construct such a system, ferrocene-containing redox mediators (GOX-pFcAc) were covalently coupled to glucose oxidase. Then, using the chitosan, human serum albumin (HAS) and GOX-pFcAc were drop cast onto carbon paper strips. Chit-GOX-pFcAcHSA-carbon paper biosensor yielded a glucose sensitivity of $0.33 \mu \mathrm{A} /\left(\mathrm{mM} \cdot \mathrm{cm}^{2}\right)$. The detailed studies were summarized for comparison in Table 3.

One of the most crucial issues is trying to avoid interference effect of other compounds found in the matrix for real samples. For this reason, selected compounds $(0.5 \mathrm{mM})$ were tested. To test their effects, they were injected to the reaction cell instead of glucose. Their amperometric responses were recorded and depicted in Figure 8. According to the results, no interference effect on biosensors was observed during measurements. When glucose was injected in 
Table 3. Analytical properties of conjugated polymer based enzymatic glucose biosensors with different electrodes.

\begin{tabular}{|c|c|c|c|c|c|}
\hline & $\begin{array}{c}K_{\mathrm{M}}^{\mathrm{app}} \\
{[\mathrm{mM}]}\end{array}$ & $\begin{array}{l}I_{\max } \\
{[\mu \mathbf{A}]}\end{array}$ & $\begin{array}{c}\text { LOD } \\
{[\mathrm{mM}]}\end{array}$ & $\begin{array}{c}\text { Sensitivity } \\
{\left[\mu \mathbf{A} /\left(\mathbf{m M} \cdot \mathbf{c m}^{2}\right)\right]}\end{array}$ & Ref. \\
\hline $\begin{array}{l}\mathrm{Pt} / \mathrm{GOx}-\mathrm{PA} \\
\mathrm{Pt} / \mathrm{PB} / \mathrm{GOx}-\mathrm{PAn}\end{array}$ & 28.800 & $\begin{array}{l}0.440 \\
0380\end{array}$ & $\begin{array}{l}\mathrm{NR} \\
\mathrm{NRC}\end{array}$ & $\begin{array}{r}1.500 \\
300\end{array}$ & [12] \\
\hline $\begin{array}{l}\mathrm{Pt} / \mathrm{PB} / \mathrm{GUx}-\mathrm{PAn} \\
\mathrm{Pt} / \mathrm{PB} / \mathrm{GOx}-\mathrm{PPy}\end{array}$ & $\begin{array}{l}10.300 \\
17.300\end{array}$ & $\begin{array}{l}0.380 \\
0.110\end{array}$ & $\begin{array}{c}\mathrm{NKC} \\
\mathrm{NR}\end{array}$ & $\begin{array}{r}3.00 \\
1.300\end{array}$ & {$[14]$} \\
\hline PPy-GOx/PPy-Cl & 8.400 & 1.540 & 0.027 & 3.500 & [42] \\
\hline GOx-RGO/Nafion & NR & NR & 0.170 & 3.800 & [44] \\
\hline Poly(adamantanepyrrole)/SWCNT/ $\beta$-cyclodextrin/GOx & 5.000 & NR & NR & 31.020 & [45] \\
\hline Poly(BEDOA-6)/AuNPs/MPA/GOx & 0.810 & 7.100 & 0.025 & 14.970 & [46] \\
\hline PFLO/AuNPs/graphene/GOx & 0.229 & 2.198 & 0.081 & 7.3570 & [47] \\
\hline PTTzTh/GOx & 0.970 & 4.820 & 0.075 & 36.320 & This work \\
\hline
\end{tabular}

the medium, a sharp and quick response was observed contrary to the case for interfering compounds. The proposed sensor was applied for quantitative analysis of glucose in beverages. Real samples were added to buffer instead of analyte and glucose concentration was calculated from the linear graph obtained by calibration curve. Real samples were directly used without any pre-treatment. The glucose content of the $\mathrm{L}^{\circledR}$ ice tea was calculated as $0.387 \mathrm{mM}$ for PTTzTh/GOx sensor and as $0.38 \mathrm{mM}$ using reference method (1.84\% error). For a sample of $\mathrm{C}^{\circledR}$ cherry juice, glucose content was calculated as $0.63 \mathrm{mM}$ for PTTzTh/GOx sensor and $0.72 \mathrm{mM}$ for reference method $(-12.5 \%$ error). It was concluded that glucose detection in the present samples can be performed even the presence of other interfering compounds using the proposed biosensor system. Cyclic voltammetry studies were performed to investigate the changes in the effective surface area following the deposition of each additional layer. In this experiment, three different electrodes were prepared as follows: a bare electrode, PTTzTh and PTTzTh/

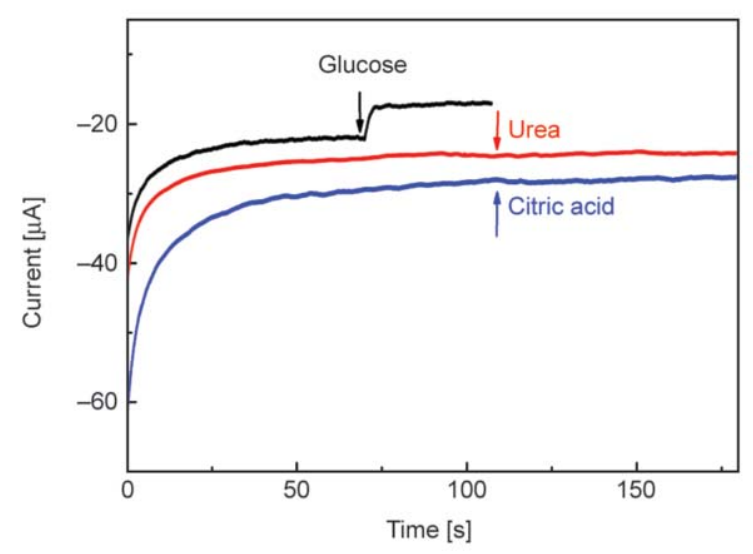

Figure 8. Effect of some interfering compounds and glucose to current (in $50 \mathrm{mM}$ phosphate buffer $\mathrm{pH} 7.0$ buffer, $\left.25^{\circ} \mathrm{C},-0.7 \mathrm{~V}\right)$.
GOx. Experiments were carried out in a solution containing $5 \mathrm{mM} \mathrm{Fe}(\mathrm{CN})_{6}^{3-/ 4}, 0.1 \mathrm{M} \mathrm{KCl}$ and $\mathrm{pH}$ 7.0 phosphate buffer at potentials between -0.4 and $0.8 \mathrm{~V}$. Figure 9 shows the voltammograms of three different electrodes modified with; PTTzTh and PTTzTh/GOx under optimum conditions and the results were compared with an unmodified electrode. The measured anodic current peaks $\left(I_{\mathrm{p}}\right)$ were found to be for bare graphite electrode: $0.12 \mathrm{~mA}$, for graphite electrode/PTTzTh: $0.26 \mathrm{~mA}$ and for graphite electrote/PTTzTh /GOx: $0.195 \mathrm{~mA}$. Average electroactive surface areas were calculated using RandlesSevcik equation (Equation (1)) [48]:

$I_{\mathrm{p}}=2.69 \cdot 10^{5} A D^{1 / 2} n^{3 / 2} v^{1 / 2} C$

According to the Equation (1), electroactive surface areas of the electrodes (bare, PTTzTh and PTTzTh/ GOx) were estimated as $0.106,0.235$ and $0.174 \mathrm{~cm}^{2}$, respectively. As seen in Figure 9, results clearly showed that a polymer modified electrode offers higher effective surface area compared to bare electrode

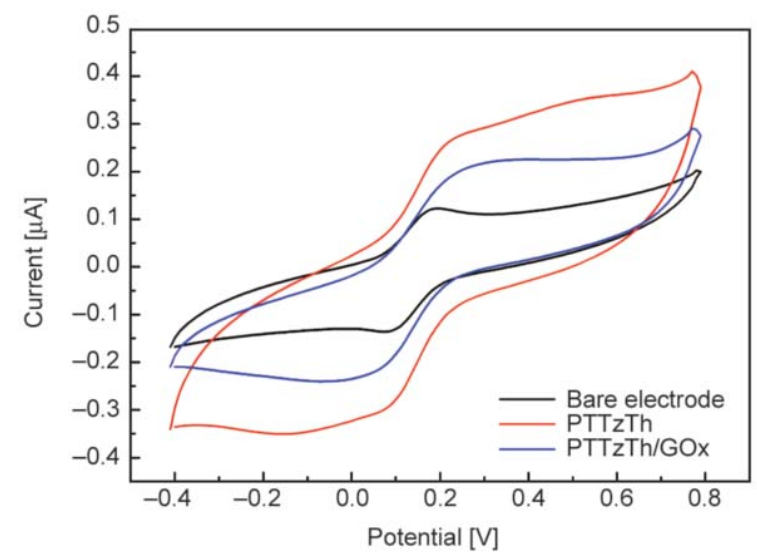

Figure 9. Cyclic voltammograms of bare electrode, PTTzTh and PTTzTh/GOx in $5 \mathrm{mM} \mathrm{Fe}(\mathrm{CN})_{6}{ }^{3-/ 4-}$ containing $0.1 \mathrm{M} \mathrm{KCl}$ and $\mathrm{pH} 7.0$ phosphate buffer at $100 \mathrm{mV} / \mathrm{s}$. 


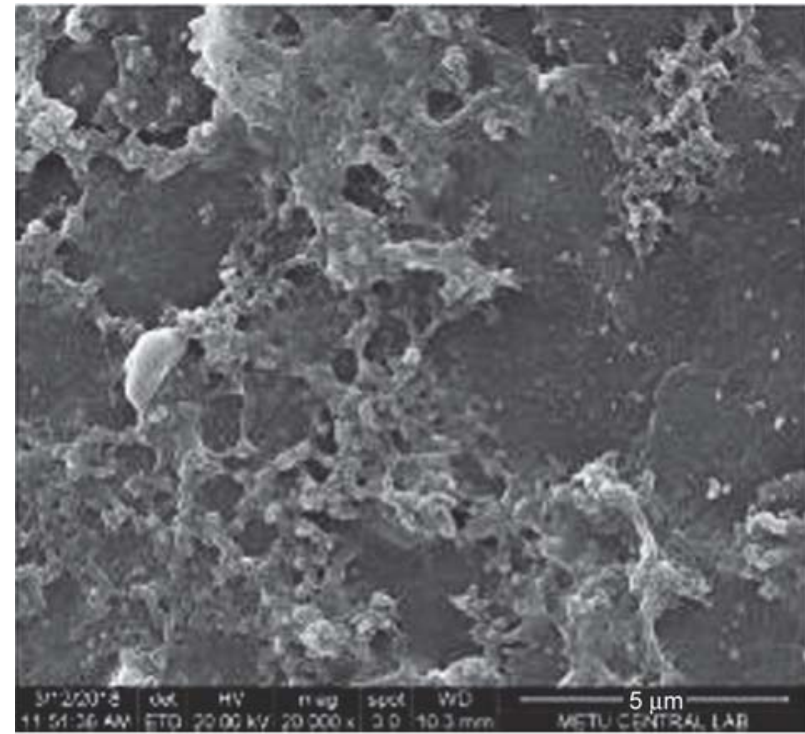

a)

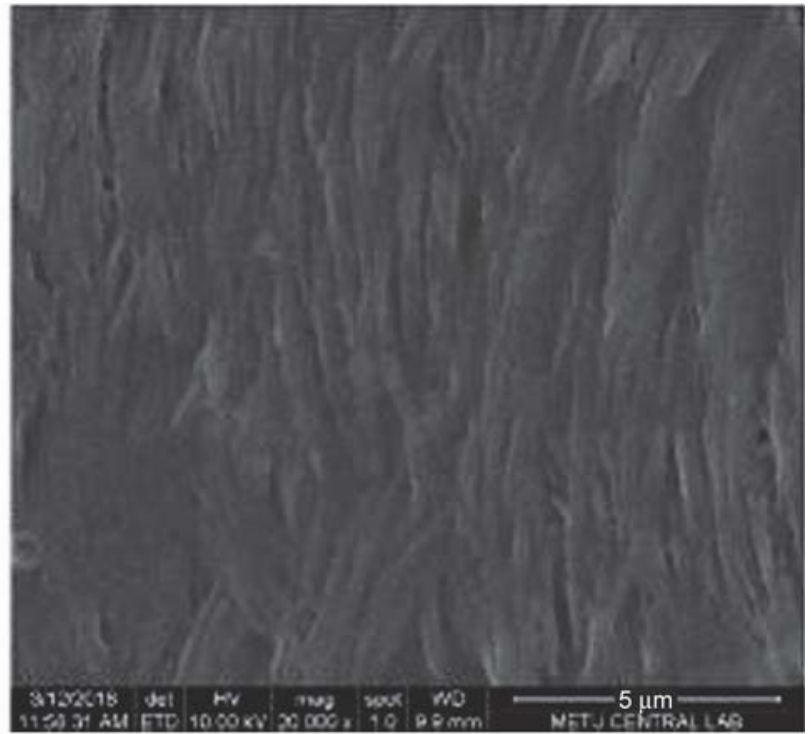

b)

Figure 10. SEM images of PTTzTh (a) and (b) PTTzTh/GOx under optimum conditions.

indicating the contribution of the polymers to charge transfer. Successive formation of the layers on the transducer surface alters the interfacial structures of the surfaces. After the biomolecule deposition, a slight decrease in the effective surface area was observed. This is due to the presence of the additional insulator layer on the surface.

The surface morphologies of modified electrodes during stepwise modification were characterized by the scanning electron microscopy (SEM). Figure 10 depicts SEM images of PTTzTh and PTTzTh/GOx modified electrodes. In Figure 10a, the well-known cauliflower structure of the conducting polymers was observed. Different surface properties were observed after biomolecule deposition on polymer coated surface (Figure 10b). Obvious changes in surface morphologies were evidences for successful surface modifications.

\section{Conclusions}

In this work, we have synthesized two thiazolothiazole based donor-acceptor-donor type polymers. Optical and electrochemical properties of the polymers were investigated. The incorporation of methyl unit into the conjugated backbone causes steric hindrance, resulting in less order and consequently increasing the $E_{\mathrm{g}}$ of the PTTzMTh. Moreover, biosensor application of PTTzTh was performed to detect glucose in beverages successfully. Under the optimized conditions, analytical and kinetic characteristics of the system were analyzed and the sensor exhibited promising biosensor characteristics. To the best of our knowledge electrochemical properties of the proposed polymers were for the first time investigated and showed promising electrochromic properties. Additionally, the development of the PTTzTh/GOxmodified graphite electrode is simple and involves a single step procedure without using additional materials that offers a great advantage for glucose biosensing system.

\section{References}

[1] Pal N., Saha B., Kundu S. K., Bhaumik A., Banerjee S.: A highly efficient non-enzymatic glucose biosensor based on a nanostructured $\mathrm{NiTiO}_{3} / \mathrm{NiO}$ material. New Journal of Chemistry, 39, 8035-8043 (2015). https://doi.org/10.1039/C5NJ01341K

[2] Wang J.: Electrochemical glucose biosensors. Chemical Reviews, 108, 814-825 (2008). https://doi.org/10.1021/cr068123a

[3] Tiwari A., Shukla S. K.: Chitosan-g-polyaniline: A creatine amidinohydrolase immobilization matrix for creatine biosensor. Express Polymer Letters, 3, 553-559 (2009).

\section{https://doi.org/10.3144/expresspolymlett.2009.69}

[4] Kausaite-Minkstimiene A., Simanaityte R., Ramanaviciene A., Glumbokaite L., Ramanavicius A.: Reagentless amperometric glucose biosensor based on a graphite rod electrode layer-by-layer modified with 1,10-phenanthroline-5,6-dione and glucose oxidase. Talanta, 171, 204-212 (2017). https://doi.org/10.1016/j.talanta.2017.04.047

[5] Wu C. M., Yu S. A., Lin S. L.: Graphene modified electrospun poly(vinyl alcohol) nanofibrous membranes for glucose oxidase immobilization. Express Polymer Letters, 8, 565-573 (2014).

https://doi.org/10.3144/expresspolymlett.2014.60 
[6] Wang J., Musameh M., Lin Y.: Solubilization of carbon nanotubes by nafion toward the preparation of amperometric biosensors. Journal of American Chemical Society, 125, 2408-2409 (2003).

https://doi.org/10.1021/ja028951v

[7] Kros A., van Hövell S. W. F. M., Sommerdijk N. A. J. M., Nolte R. J. M.: Poly(3,4-ethylenedioxythiophene)based glucose biosensors. Advanced Materials, 13, 1555-1557 (2001).

https://doi.org/10.1002/1521-4095(200110)13:20<1555::AIDADMA1555>3.0.CO;2-7

[8] Ensafi A. A., Khoddami E., Rezaei B.: Aptamer@AuO-phenylenediamine modified pencil graphite electrode: A new selective electrochemical impedance biosensor for the determination of insulin. Colloids and Surfaces B: Biointerfaces, 159, 47-53 (2017). https://doi.org/10.1016/j.colsurfb.2017.07.076

[9] Cosnier S.: Biomolecule immobilization on electrode surfaces by entrapment or attachment to electrochemically polymerized films. A review. Biosensensors and Bioelectronics, 14, 443-456 (1999). https://doi.org/10.1016/S0956-5663(99)00024-X

[10] Soganci T., Soyleyici H. C., Demirkol D. O., Ak M., Timur S.: Use of super-structural conducting polymer as functional immobilization matrix in biosensor design. Journal of the Electrochemical Society, 265, B22B26 (2018).

https://doi.org/10.1149/2.0561802jes

[11] Al-Mokaram A. M. A. A. A., Yahya R., Abdi M. M., Mahmud H. N. M. E.: One-step electrochemical deposition of polypyrrole-chitosan-iron oxide nanocomposite films for non-enzymatic glucose biosensor. Materials Letters, 183, 90-93 (2016).

https://doi.org/10.1016/j.matlet.2016.07.049

[12] Garjonyte R., Malinauskas A.: Amperometric glucose biosensors based on prussian blue- and polyanilineglucose oxidase modified electrodes. Biosensensors and Bioelectronics, 15, 445-451 (2000). https://doi.org/10.1016/S0956-5663(00)00101-9

[13] Zhai D., Liu B., Shi Y., Pan L., Wang Y., Li W., Zhang R., Yu G.: Highly sensitive glucose sensor based on Pt nanoparticle/polyaniline hydrogel heterostructures. ACS Nano, 7, 3540-3546 (2013). https://doi.org/10.1021/nn400482d

[14] Garjonyte R., Malinauskas A.: Glucose biosensor based on glucose oxidase immobilized in electropolymerized polypyrrole and $\operatorname{poly}(o$-phenylenediamine $) /$ films on a Prussian Blue-modified electrode. Sensors and Actuators B: Chemical, 63, 122-128 (2000). https://doi.org/10.1016/S0925-4005(00)00317-8

[15] Osaka I., Zhang R., Sauvé G., Smilgies D-M., Kowalewski T., McCullough R. D.: High-lamellar ordering and amorphous-like $\pi$-network in short-chain thiazolothiazole-thiophene copolymers lead to high mobilities. Journal of American Chemical Society, 131, 2521-2529 (2009). https://doi.org/10.1021/ja801475h
[16] Osaka I., Sauvé G., Zhang R., Kowalewski T., McCullough R. D.: Novel thiophene-thiazolothiazole copolymers for organic field-effect transistors. Advanced Materials, 19, 4160-4165 (2007). https://doi.org/10.1002/adma.200701058

[17] Subramaniyan S., Xin H., Kim F. S., Jenekhe S. A.: New thiazolothiazole copolymer semiconductors for highly efficient solar cells. Macromolecules, 44, 62456248 (2011). https://doi.org/10.1021/ma201054a

[18] Lim D-H., Jang S-Y., Kang M., Lee S., Kim Y-A, Heo Y-J., Lee M-H., Kim D-Y.: A systematic study on molecular planarity and D-A conformation in thiazolothiazole- and thienylenevinylene-based copolymers for organic field-effect transistors. Journal of Material Chemistry C, 5, 10126-10132 (2017). https://doi.org/10.1039/C7TC02273E

[19] Woodward A. N., Kolesar J. M., Hall S. R., Saleh N-A., Jones D. S., Walter M. G.: Thiazolothiazole fluorophores exhibiting strong fluorescence and viologen-like reversible electrochromism. Journal of American Chemical Society, 139, 8467-8473 (2017).

https://doi.org/10.1021/jacs.7b01005

[20] Wilson R., Turner A. P. F.: Glucose oxidase: An ideal enzyme. Biosensors and Bioelectronics, 7, 165-185 (1992). https://doi.org/10.1016/0956-5663(92)87013-F

[21] Jung I. H., Yu J., Jeong E., Kim J., Kwon S., Kong H., Lee K., Woo H. Y., Shim H-K.: Synthesis and photovoltaic properties of cyclopentadithiophene-based lowbandgap copolymers that contain electron-withdrawing thiazole derivatives. Chemistry A European Journal, 16, 3743-3752 (2010). https://doi.org/10.1002/chem.200903064

[22] Wang B., Zhao J., Cui C., Liu J., He Q.: Electrosynthesis and characterization of an electrochromic material from poly(1,4-bis(3-methylthiophen-2-yl)benzene) and its application in electrochromic device. Solar Energy Materials and Solar Cells, 98, 161-167 (2012). https://doi.org/10.1016/j.solmat.2011.10.032

[23] Yue R., Xu J., Lu B., Liu C., Li Y., Zhu Z., Chen S.: Electrochemical copolymerization of benzanthrone and 3-methylthiophene and characterization of their fluorescent copolymer. Journal of Material Science, 44, 5909-5918 (2009). https://doi.org/10.1007/s10853-009-3836-6

[24] Xu Z., Wang M., Zhao J., Cui C., Fan W., Liu J.: Donor-acceptor type neutral green polymers containing 2,3-di(5-methylfuran-2-yl) quinoxaline acceptor and different thiophene donors. Electrochimica Acta, 125, 241-249 (2014). https://doi.org/10.1016/j.electacta.2013.12.097 
[25] Chen S., Zhang D., Wang M., Kong L., Zhao J.: Donoracceptor type polymers containing the 2,3-bis(2-pyridyl)5,8-dibromoquinoxaline acceptor and different thiophene donors: Electrochemical, spectroelectrochemistry and electrochromic properties. New Journal of Chemistry, 40, 2178-2188 (2016).

https://doi.org/10.1039/C5NJ02651B

[26] Ming S., Zhen S., Lin K., Zhao L., Xu J., Lu B.: Thiadiazolo[3,4-c]pyridine as an acceptor toward fast-switching green donor-acceptor-type electrochromic polymer with low bandgap. ACS Applied Materials and Interfaces, 7, 11089-11098 (2015).

https://doi.org/10.1021/acsami.5b01188

[27] Roncali J.: Conjugated poly(thiophenes): Synthesis, functionalization, and applications. Chemical Reviews, 92, 711-738 (1992).

https://doi.org/10.1021/cr00012a009

[28] Chen W., Xue G.: Low potential electrochemical syntheses of heteroaromatic conducting polymers in a novel solvent system based on trifluroborate-ethyl ether. Progress in Polymer Science, 30, 783-811 (2005). https://doi.org/10.1016/j.progpolymsci.2005.03.002

[29] Roth S., Bleier H.: Solitons in polyacetylene. Advances in Physics, 36, 385-462 (1987). https://doi.org/10.1080/00018738700101032

[30] Zhao H., Tang D., Zhao J., Wang M., Dou J.: Two novel ambipolar donor-acceptor type electrochromic polymers with the realization of RGB (red-green-blue) display in one polymer. RSC Advances, 4, 61537-61547 (2014). https://doi.org/10.1039/C4RA11628C

[31] Gunbas G., Toppare L.: Electrochromic conjugated polyheterocycles and derivatives-highlights from the last decade towards realization of long lived aspirations. Chemical Communications, 48, 1083-1101 (2012). https://doi.org/10.1039/C1CC14992J

[32] Lv X., Li W., Ouyang M., Zhang Y., Wrigh D. S., Zhang C.: Polymeric electrochromic materials with donor-acceptor structures. Journal of Material Chemistry C, 5, 12-28 (2017).

https://doi.org/10.1039/C6TC04002K

[33] Periasamy A. P., Chang Y-J., Chen S-M.: Amperometric glucose sensor based on glucose oxidase immobilized on gelatin-multiwalled carbon nanotube modified glassy carbon electrode. Bioelectrochemistry, 80, 114-120 (2011).

https://doi.org/10.1016/j.bioelechem.2010.06.009

[34] Wan D., Yuan S., Li G. L., Neoh K. G., Kang E. T.: Glucose biosensor from covalent immobilization of chitosan-coupled carbon nanotubes on polyaniline-modified gold electrode. ACS Applied Materials Interfaces, 2, 3083-3091 (2010).

https://doi.org/10.1021/am100591t
[35] Garjonyte R., Malinauskas A.: Glucose biosensor based on glucose oxidase immobilized in electropolymerized polypyrrole and poly (o-phenylenediamine) films on a Prussian Blue-modified electrode. Sensors and Actuators B: Chemical, 63, 122-128 (2000).

https://doi.org/10.1016/S0925-4005(00)00317-8

[36] Shi Q., Li Q., Shan D., Fan Q., Xue H.: Biopolymerclay nanoparticles composite system (chitosan-laponite) for electrochemical sensing based on glucose oxidase. Materials Science and Engineering C, 28, 1372-1375 (2008). https://doi.org/10.1016/j.msec.2008.03.001

[37] Holzinger M., Bouffier L., Villalonga R., Cosnier S.: Adamantane/ $\beta$-cyclodextrin affinity biosensors based on single-walled carbon nanotubes. Biosensors and Bioelectronics, 24, 1128-1134 (2009). https://doi.org/10.1016/j.bios.2008.06.029

[38] Pan D., Chen J., Nie L., Tao W., Yao S.: An amperometric glucose biosensor based on poly( $o$-aminophenol) and prussian blue films at platinum electrode. Analytical Biochemistry, 324, 115-122 (2004). https://doi.org/10.1016/j.ab.2003.09.029

[39] Alim S., Kafi A. K. M., Rajan J., Yusoff M. M.: Application of polymerized multiporous nanofiber of $\mathrm{SnO}_{2}$ for designing a bienzyme glucose biosensor based on HRP/GOx. International Journal of Biological Macromolecules, 123, 1028-1034 (2019).

https://doi.org/10.1016/j.ijbiomac.2018.11.171

[40] Phetsang S., Jakmunee J., Mungkornasawakul P., Laocharoensuk R., Ounnunkad K.: Sensitive amperometric biosensors for detection of glucose and cholesterol using a platinum/reduced graphene oxide/poly(3aminobenzoic acid) film-modified screen-printed carbon electrode. Bioelectrochemistry, 127, 125-135 (2019). https://doi.org/10.1016/j.bioelechem.2019.01.008

[41] Al-Sagur H., Komathi S., Karakaş H., Atilla D., Gürek A. G., Basova T., Farmilo N., Hassan A. K.: A glucose biosensor based on novel Lutetium bis-phthalocyanine incorporated silica-polyaniline conducting nanobeads. Biosensors and Bioelectronics, 102, 637-645 (2018). https://doi.org/10.1016/j.bios.2017.12.004

[42] Ayenimo J. G., Adeloju S. B.: Amperometric detection of glucose in fruit juices with polypyrrole-based biosensor with an integrated permselective layer for exclusion of interferences. Food Chemistry, 229, 127-135 (2017). https://doi.org/10.1016/j.foodchem.2017.01.138

[43] Campbell A. S., Islam M. F., Russell A. J.: Intramolecular electron transfer through poly-ferrocenyl glucose oxidase conjugates to carbon electrodes: 1 . Sensor sensitivity, selectivity and longevity. Electrochimica Acta, 248, 578-584 (2017). https://doi.org/10.1016/j.electacta.2017.07.150

[44] Choi B. G., Im J., Kim H. S., Park H.: Flow-injection amperometric glucose biosensors based on graphene/ nafion hybrid electrodes. Electrochimica Acta, 56, 9721-9726 (2011). https://doi.org/10.1016/j.electacta.2011.07.118 
[45] Holzinger M., Bouffier L., Villalonga R., Cosnier S.: Adamantane/ $\beta$-cyclodextrin affinity biosensors based on single-walled carbon nanotubes. Biosensors and Bioelectronics, 24, 1128-1134 (2009).

https://doi.org/10.1016/j.bios.2008.06.029

[46] Kesik M., Kocer O., Kanik F. E., Unlu N. A., Rende E., Evren A-G., Rossi R. M., Udum Y. A., Toppare L.: Effective and functional surface design for biosensing applications based on a novel conducting polymer and PMMA/clay nanocomposite. Electroanalysis, 25, 1995 2006 (2013).

https://doi.org/10.1002/elan.201300193
[47] Gokoglan T. C., Kesik M., Soylemez S., Yuksel R., Unalan H. E., Toppare L.: Paper based glucose biosensor using graphene modified with a conducting polymer and gold nanoparticles. Journal of the Electrochemical Society, 164, G59-G64 (2017).

https://doi.org/10.1149/2.0791706jes

[48] Bard A. J., Faulkner L. R.: Electrochemical methods: Fundamentals and applications. Wiley, New York (2000). 